\title{
Translation Quality Assessment in Relevance Theory
}

$$
\text { Zhuo WANG }{ }^{\mathrm{a}} \text { and Yu WANG }{ }^{\mathrm{b}, *}
$$

\author{
Foreign Languages Department, Tianjin University of Finance and Economics, Tianjin, China \\ awangzhuoxy@163.com, bbonnieangel@163.com \\ ${ }^{\star}$ Corresponding Author
}

\section{Keywords: Relevance Theory, Translation, Quality Assessment}

\begin{abstract}
There is few criterion of translation quality assessment in the light of relevance theory. With the help of comparative analysis approach and literature review method, this paper explores the development of relevance theory, analyzes the relationship between relevance theory and translation, and explains why optimal relevance can become a standard of translation quality assessment. The result shows that relevance theory does have the guiding effect on most translation phenomenons, but lacks of the explanatory power towards literary translation.
\end{abstract}

\section{Introduction}

Relevance theory is a very influential theory of cognitive pragmatics in the west recent years. In 1986, French Sperber Dan and Britain Wilson coauthored a book named Relevance: Cognition and Communication, in which relevance theory formally comes into being. Shen Jiaxuan was the first people who brought relevance theory to China. He published a paper in 1988, in which the book Relevance: Cognition and Communication was introduced. Therefore, relevance theory entered linguistic field in China[1]. Although translation is a complicated phenomenon, it can still gets lots of useful enlightenment from relevance theory, for translation is also a kind of communicative activity substantially.

\section{Conversational Implicature VS Relevance Theory}

When it comes to relevance theory, conversational implicature is the first should be mentioned. American philosopher and linguist H.P.Grice is the founder of conversational implicature, and he has created another important area of research for language learning. According to Grice[2], people should obey some basic principles in order to make the conversation go on, especially "cooperative principle”. And what does conversational implicature have to do with relevance theory? In fact, relevance theory is a pragmatic theory proposed for cooperative principle of conversational implicature, not only for its simple development and inheritance, but also a kind of supplement and discard.

\section{Conversational Implicature}

People's discourse communication is always mutual-cooperative, because both parties want their own words to be understood by the other party. Therefore both parties will abide by certain cooperative principles to make communication successful in the process of communicating. Mr. Grice believes that people can create high-quality and productive conversations if they are able to stick to cooperative principle in communication. To this end, he refined out of four principles that people should follow when communicating under the framework of cooperative principle. The first one is The Maxim of Quantity, which means to be truthful. Do not say what you believe to be false and that for which you lack adequate evidence. The second one is The Maxim of Quality, which means quality of information. Make your contribution as informative as required and do not make your contribution more informative than it is required. The third one is The Maxim of Relation, which means to be relevant. The last one is The Maxim of Manner, which means to be orderly and 
brief. Obscurity of expression and ambiguity should be avoided[3]. In other words, according to Grice's idea, as long as people adhere to these guidelines in communicating, they can achieve the purpose of a successful communication. However, people do not always strictly abide by cooperative principle in actual verbal communication, sometimes even deliberately violate it. Mr. Grice called this situation "special conversational implicature", meaning that it is ostensibly violating cooperative principle. That is to say, what the "special conversational implicature" usually express are not the literal meaning of the word itself, but the deeper meaning that the speaker wants to express to the listener.

\section{Relevance Theory}

According to Gutt[4], the central claim of relevance theory is that human communication crucially creates an expectation of optimal relevance, that is, an expectation on the part of the audiences that his attempt at interpretation will yield adequate contextual effects at minimal processing cost. Why does relevance theory emphasize the expectation of optimal relevance instead of maximal relevance? When the listener get the maximum contextual effects with the minimum processing efforts, it is no doubt that he or she gets maximal relevance. However, maximal relevance is not what people pursuing sometimes. In order to make a successful and efficiently communication, the listener only needs to get adequate contextual effects to understand what the speaker said, which means the listener does not need to get unnecessary contextual effects with unnecessary processing efforts. Therefore, the strength of relevance depends on two factors: processing effort and contextual effect. On even ground, the fewer processing efforts are, the stronger relevance is; the more contextual effects are, the stronger relevance is. As a matter of fact, there is a potential context in everyone's brain, which stores plenty of information. However, when there is a particular occasion, not all information can be retrieved with the same processing efforts. The listener uses minimal processing efforts to obtain adequate contextual effects, which is to obtain optimal relevance. In short, the core of relevance theory is to produce optimal relevance. That is to say, the listener can get adequate contextual effects with justifiable processing efforts in verbal understanding. Each ostensive-inferential process behavior is assumed to have its own optimal relevance: the listener expects to get adequate contextual effects without the extra processing efforts.

An example is taken to explain: a boy cried at home after school, his father bought him a new bag, then the boy stopped crying. A conclusion can be drawn from this example, that is there are some links between "crying" and "new bag", otherwise why this father does not buy other things to his son, or how does the father know that a new bag can stop his son from crying? According to relevance theory, "seeking relevance" in the process of communicating is mainly depends on the analysis of three kinds of information: old information, new information and related information. Old information is the information known to the topic, new information is the information unknown to the topic, and related information is the information links the old and new information together. In communicating, the roles of the three are different, but related to each other: the old information is the premise of communication understanding, the new information is understood based on the old information, and the related information plays an important role in accelerating the communication speed. In addition, all kinds of information have different functions to communicators. Some information always attracts the communicator's attention more than others, while the communicator is always the first to understand and deal with the information that catches his or her attention. Under the framework of relevance theory, the relationship between the information that communicator faced and the effects that given to the communicator is called relevance relation. Relevance relation is of vital importance in communicating. From the perspective of the speaker, the utterance should be reasonable and consistent, so the language should be concise and clear; from the perspective of the listener, the understanding of the utterance must be based on the speaker's words. Thus, the understanding of utterance depends on reasoning, and the process of reasoning is to find relevance. Only by finding the relevance of words correctly can the communication succeed. 


\section{Relevance Theory of Translation}

So what's the connection between relevance theory and translation? How does relevance theory guide translation? Modern translators believe that translation is a process of communicating between the author, the translator and the reader. Both translators and readers should understand the meanings expressed by the original author in the source text, especially translators. According to relevance theory, there are two meanings in communication, one is explicature, the other is implicature. Communicators should understand not only the literal meaning of the utterance, but also something behind it. Therefore, under the framework of relevance theory, translation can provide the target language readers with adequate contextual effect to achieve optimal relevance and realize mutual manifestness.

With the deepening of the research, scholars have combined relevance theory and translation together gradually. Ernst August Gutt published a thesis named Cognition and Relevance, and in 1991, he published this paper as a book named Translation and Relevance: Cognition and Context, in which relevance theory is firstly applied to translation research, bringing a deep influence to translation field. Gutt held the view that translation should be the acceptable target language which have the same meaning with the source language. Optimal relevance is not only a goal that all translators striving for, but also a criterion of translation quality assessment. The translator's duty is to try to match the intention of the original author with the expectation of the target audience.

\section{Optimal Relevance}

Relevance is the basis of cognition, that is to say, cognition is based on the acquisition of relevance. Like any other concepts, there is also a criterion in relevance theory. As it mentioned above, optimal relevance comes from relevance theory, which means that the listener can obtain adequate contextual effects without unnecessary processing efforts when inferring the speaker's explicature. Optimal relevance brings a new thinking to the translation field: translators can use all available translation methods and techniques in order to let readers acquire the real intention of the author with justifiable processing efforts.

According to Ma Hui[5], different translators have different views about translation, sometimes their views are consistent with others, while sometimes are contradictory. However, relevance theory can provide a unified theoretical framework to translations, and also offer a definite answer to queries about all kinds of translation activities. Indeed, relevance theory differs from other translation theories. It analyzes translation from a macro perspective. Some translation theories believe that translation should like the source language, but some hold the view that translation should like the target language; some translation theories argue that translation can be added or decreased appropriately, while some insist that methods of adding and omitting is not allowed. Each of these claim seems reasonable, thus the contention of different schools of translation has not stopped for many years. However, within the framework of relevance theory, these problems would no longer exist, because translation is a communicative behavior in essence, thus optimal relevance becomes the only standard of translation quality assessment, as previously mentioned. In other words, all those translations that convey optimal relevance to the readers can be called good translations, no matter what translation strategies are used. There is not a completely unified theory to show how the good translation is formed, should it looks like source language or target language, for different styles of the source language may use different translation methods and skills.

\section{Ostensive-inferential Activity}

Gutt regarded translation as an inferential process which involved in brain mechanism. As previously mentioned, translation is an ostensive-inferential activity. To get a successful communication, reasonable interpretation should be made in order to achieve optimal relevance. This process involves in dynamic inference of dynamic context, while people's inference is based on relevance instead of other standards or principles. Since translation is a kind of communication, understandings of source text and how to choose codes during translation are also based on relevance. Therefore, seeking relevance becomes the goal that translators striving to achieve, and 
also becomes the criterion for judging the quality of translation. In other words, a good translation can establish optimal relevance between the author and the readers, so that the readers can get the intention of the author. This is a high requirement for translators, because translators should not only fully understand the author's intention themselves, but also consider the reader's cognitive environment, so that can convey the author's intention to the readers in a good way.

\section{Cognitive Environment}

In addition, the reader's cognitive environment is also one of the important factors to be considered. If the reader has the same or similar concept as the original writer in the cognitive environment, then reproducing the style of the original text will be a faithful representation undoubtedly; on the contrary, if the cognitive environment of the target language reader lacks the similar concept described by the original author, the most important thing for the translator is to make the reader understand what the original author is expressing, and then try to be faithful to the original text in language form as much as possible. According to Jia Yan and Yang Shuai[6], the cognitive environment of a person can reflect the communicative cues, and the different cognitive environment between the sender and the receiver will lead to the difference of discourse correlation. Therefore, the translator should be integrated into the cognitive environment of the sender, and know the cultural default in the language, so to understand the explicature and implicature of the sender correctly.

\section{Limitations of Relevance Theory of Translation}

However, some scholars have questioned the universality of relevance theory of translation. He Ziran and Ran Yongping believed that contextual effect and processing effort are two factors that restrict the relationship. However, through relevance theory, contextual effect that the listener wants to get determines how much processing efforts he or she would spend, while it is also determined by the degree of the processing effort in turn. Therefore, it is hard to say which one is the decider. This contradiction makes it difficult for the translator to grasp the relationship between the processing effort and the contextual effect so as to make it difficult to measure the correlation degree[7]. According to Chen Yumin[8], culture default is inevitable in the framework of relevance theory, but the contents of culture default can not be found in the passage. Thus, translator's knowledge and experience can not be connected well, which will make it difficult for readers to understand.

\section{Summary}

Although relevance theory does not have sufficient explanatory power for literary translation, the contribution of relevance theory to translation is still undeniable. Translation itself is not a perfect behavior, for there must be something missing in back translation. Therefore, how can we ask for a criterion that is suitable for all translation activities? The emergence of relevance theory of translation has explained the criterion of translation quality from a new perspective, which promoted the development of the translation field. Although translation is an extremely complex phenomenon, it can still get a lot of useful enlightenment from relevance theory, which is probably the greatest contribution of relevance theory to translation.

\section{Acknowledgment}

This work was financially supported by Pre-research of Foundation for The Youth Scholars of Tianjin University of Finance and Economics (05010161), China.

\section{References}

[1] Yang Chunhui, Overview on Relevance Theory of Translation, Spiritual Leaders, 2015, 96. 
[2] Grice, H.P,Logic and Conversation, In Cole and Morgan, 1975.

[3] Cooperative principle, on http://ishare.iask.sina.com.cn/f/22491498.html

[4] Gutt, E. A, Translation and relevance: Cognition and context, Basil Blackwell Ltd, 1991.

[5] Ma Hui, Translation Quality Assessment in the Light of Relevance Theory, Academic Research, 2015,38.

[6] Jia Yan, Yang Shuai, Relevance Theory and Translation Study, Journal of Mudanjiang Normal University, 2014, 110.

[7] He Ziran, Ran Yongping, Relevance Theory--the Foundation of Cognitive Pragmatics, Modern Foreign Language, 1998, 105.

[8] Chen Yumin, Explanation of Relevance Theory towards Cultural Translation, Overseas English, $2017,121$. 